MATHEMATICS OF COMPUTATION

Volume 73, Number 245, Pages 19-34

S $0025-5718(03) 01532-1$

Article electronically published on May 7, 2003

\title{
NUMERICAL SOLUTION \\ OF THE ELASTIC BODY-PLATE PROBLEM BY NONOVERLAPPING DOMAIN DECOMPOSITION TYPE TECHNIQUES
}

\author{
JIANGUO HUANG
}

\begin{abstract}
The purpose of this paper is to provide two numerical methods for solving the elastic body-plate problem by nonoverlapping domain decomposition type techniques, based on the discretization method by Wang. The first one is similar to an older method, but here the corresponding Schur complement matrix is preconditioned by a specific preconditioner associated with the plate problem. The second one is a "displacement-force" type Schwarz alternating method. At each iteration step of the two methods, either a pure body or a pure plate problem needs to be solved. It is shown that both methods have a convergence rate independent of the size of the finite element mesh.
\end{abstract}

\section{INTRODUCTION}

The mathematical modeling of elastic multi-structures, i.e., elastic structures that are assembled by elastic substructures of the same or different dimensions (three-dimensional substructures, plates, rods, etc., usually made of different elastic materials) with proper junctions, is a problem of practical importance, since such elastic structures are very common in engineering.

In the past few decades, much attention has been paid to the investigation of such elastic multi-structure problems. In [14, 15], Feng and Shi discussed the mathematical modeling of elastic multi-structures, from a mechanical and mathematical viewpoint. More recently, Ciarlet and his colleagues have made significant contributions to this field. Their main interest is the derivation of the proper junctions among substructures by the techniques of asymptotic analysis, which is a crucial step in obtaining reasonable mathematical models of elastic multi-structures. (We refer to the monograph [12] and references therein for details.) Numerical analysis on elastic multi-structures has been studied by Bernadou 2] with a conforming finite element (the Hsieh-Clough-Tocher triangle), and by Wang [24, 25] with conventional nonconforming finite elements and the TRUNC element.

In order to implement the aforementioned finite element methods efficiently, we should develop some effective solvers for the resulting linear algebraic systems.

Received by the editor December 1, 1997 and, in revised form, May 26, 2002.

2000 Mathematics Subject Classification. Primary 65N30, 65N22, 65F10, 74S05.

Key words and phrases. Nonoverlapping domain decomposition method, preconditioner, elastic multi-structures, finite element.

The work was partially supported by the National Natural Science Foundation of China under grant no. 19901018. 
Considering the special structures of these problems, the domain decomposition method seems to be a natural choice (cf. 12]). In [18, F. d'Hennezel proposed a domain decomposition method for the stiffened plate problem, discretized by the conforming finite elements. First, the global problem was transformed into a problem defined on the interface between subdomains, which led to the Schur complement matrix of the global system. Then the matrix was preconditioned by a specific operator associated only with the beam problem. Theoretical analysis and numerical examples have shown that the rate of convergence of the method does not depend on the mesh size.

In this paper, we propose two numerical methods for solving the elastic bodyplate problem by nonoverlapping domain decomposition type techniques. As in [24], we will use the trilinear conforming elements in the body part, and the bilinear conforming elements for the tangential displacements and the Adini element for the vertical displacement in the plate part. The first method is similar to the one studied in [3, 5, 18], but here the corresponding Schur complement matrix is preconditioned by a specific preconditioner associated with the plate problem. The second one, motivated by [19, 21] to some extent, is a "displacement-force" type Schwarz alternating method. The intuitive description is as follows. Given arbitrarily a guess displacement field on the interface linking the body part and the plate part, we first compute the resulting displacement field in the body part by solving a certain three-dimensional purely elastic problem which governs the deformation of the body part. Thus the stress field related to this displacement field is available on the interface. We next compute the displacement field on the plate part by solving a purely plate problem which governs the deformation of the plate part, with the aforementioned stress field as the extra "applied force" on the interface. Now we have two displacement fields on the interface: the original guess displacement and the one just obtained by computing the plate problem. We can make a weighted average of the two ones to get a new displacement field on the interface. We then use it as a new guess displacement field on the interface to implement the previous process again. We repeat the iteration until we get the desired result. We refer to (4.11)-(4.13) in Section 4 for the mathematical description of the algorithm.

A common feature of the two methods is that, at each iteration step, one needs only to solve either a pure body or a pure plate problem, which can be realized by many known efficient numerical solvers. Therefore, both methods reflect the essence of elastic multi-structures - that is, elastic multi-structures are complicated globally but their substructures are rather simple locally. On the other hand, there are some differences between the two methods. The first method needs to form the global stiffness matrix related to the elastic body-plate problem for the use of the preconditioned conjugate gradient method. The second one does not need to do so, but it requires the choice of a proper parameter to guarantee the convergence of the method.

In this paper we will estimate the rates of convergence for these two methods. With the help of Brenner's techniques (cf. 7, 8, 9]) and by some rigorous analysis, we will show that both methods have a convergence rate independent of the finite element mesh size.

The rest of this paper is organized as follows. Section 2 describes the mathematical model for the body-plate problem and its finite element approximation. 
Section 3 presents some basic results. Section 4 includes the construction of the two nonoverlapping domain decomposition methods and the estimates of their convergence rates.

\section{Mathematical model AND Finite ElEMENT APPROXimation}

For ease of exposition, let us first recall the mathematical model of the bodyplate problem and its finite element approximation. We refer to 24 for more detail. The notation reads as follows (cf. Figure 1).

$\Omega$ is the three-dimensional elastic body.

$\vec{f}^{\Omega}:=\left(f_{i}^{\Omega}\right), f_{i}^{\Omega}:=f_{i}^{\Omega}\left(x_{1}, x_{2}, x_{3}\right)$, is the applied body force in $\Omega$.

$\vec{u}^{\Omega}:=\left(u_{i}^{\Omega}\right), u_{i}^{\Omega}:=u_{i}^{\Omega}\left(x_{1}, x_{2}, x_{3}\right)$, is the displacement vector in $\Omega$.

$\omega^{t}:=\omega \times(-t, t)$ is the elastic thin plate with thickness $2 t(0<t<<1)$.

$\vec{f}^{\omega}:=\left(f_{i}^{\omega}\right), f_{i}^{\omega}:=f_{i}^{\omega}\left(x_{1}, x_{2}\right)$, is the applied surface force in $\omega$.

$\vec{u}^{\omega}:=\left(u_{i}^{\omega}\right), u_{i}^{\omega}:=u_{i}^{\omega}\left(x_{1}, x_{2}\right)$, is the displacement vector in $\omega$, with $u_{\alpha}^{\omega}=$

$0, \alpha=1,2$, and $u_{3}^{\omega}=\frac{\partial u_{3}^{\omega}}{\partial x_{2}}=0$ on $\gamma_{0}$, which means that the elastic plate is clamped on the boundary $\gamma_{0}$.

Let $\omega_{b}^{t}:=\omega_{b} \times(-t, t)$ be the intersection of the elastic body $\Omega$ and the elastic plate $\omega^{t}$. We assume that there exists a rigid junction between $\Omega$ and $\omega^{t}$, that is,

$$
\vec{u}^{\Omega}=\vec{u}^{\omega} \quad \text { on } \omega_{b} .
$$

This condition means that the displacement $\vec{u}^{\Omega}$ in the body $\Omega$ and the displacement $\vec{u}^{\omega}$ in the plate $\omega$ are continuous across the interface $\omega_{b}$.

Consider the total energy of the elastic body-plate structure under a virtual displacement vector $\vec{v}:=\left(\vec{v}^{\Omega}, \vec{v}^{\omega}\right)$ :

$$
J(\vec{v})=\frac{1}{2} D(\vec{v}, \vec{v})-F(\vec{v}),
$$

where $F(\vec{v})=F_{\Omega}\left(\vec{v}^{\Omega}\right)+F_{\omega}\left(\vec{v}^{\omega}\right), F_{\Omega}\left(\vec{v}^{\Omega}\right)=\int_{\Omega} \vec{f}^{\Omega} \cdot \vec{v}^{\Omega} d \Omega, F_{\omega}\left(\vec{v}^{\omega}\right)=\int_{\omega} \vec{f}^{\omega} \cdot \vec{v}^{\omega} d \omega$, and, for $\vec{w}=\left(\vec{w}^{\Omega}, \vec{w}^{\omega}\right)$,

$$
\begin{aligned}
D\left(\vec{v}^{\Omega}, \vec{w}^{\Omega}\right):= & \int_{\Omega} \sigma_{i j}\left(\vec{v}^{\Omega}\right) \varepsilon_{i j}\left(\vec{w}^{\Omega}\right) d \Omega+\int_{\omega} Q_{\alpha \beta}\left(\vec{v}^{\omega}\right) \varepsilon_{\alpha \beta}\left(\vec{w}^{\omega}\right) d \omega \\
& +\int_{\omega} M_{\alpha \beta}\left(\vec{v}^{\omega}\right) K_{\alpha \beta}\left(\vec{w}^{\omega}\right) d \omega,
\end{aligned}
$$
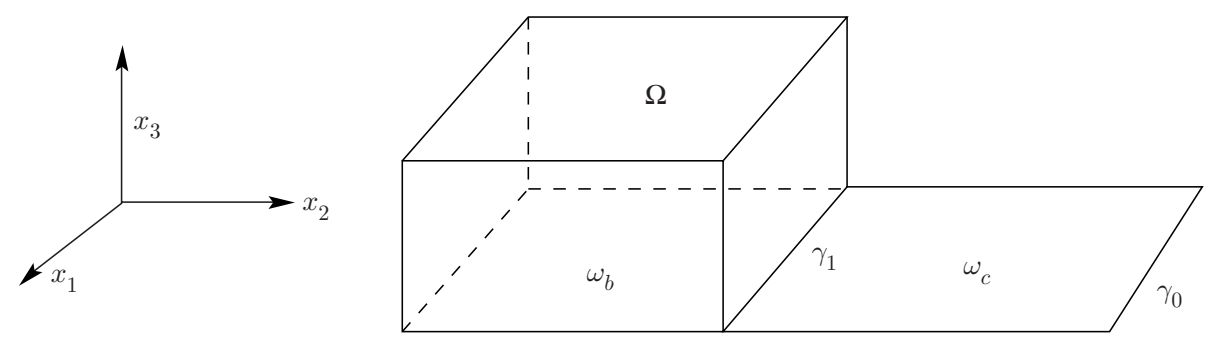

FigURE 1. 


$$
\begin{aligned}
& \varepsilon_{\alpha \beta}\left(\vec{v}^{w}\right):=\frac{1}{2}\left(\partial_{\beta} v_{\alpha}^{\omega}+\partial_{\alpha} v_{\beta}^{\omega}\right), \partial_{\alpha} v:=\frac{\partial v}{\partial x_{\alpha}}, \\
& Q_{\alpha \beta}\left(\vec{v}^{w}\right)=\frac{2 E_{\omega} \nu_{\Omega}}{1-\nu_{\omega}^{2}}\left\{\left(1-\nu_{\omega}\right) \varepsilon_{\alpha \beta}\left(\vec{v}^{w}\right)+\frac{\nu^{2}}{\left(1+\nu_{\Omega}\right)\left(1-2 \nu_{\Omega}\right)} \varepsilon_{\iota \iota}\left(\vec{v}^{w}\right) \delta_{\alpha \beta}\right\}, \alpha, \beta=1,2, \\
& K_{\alpha \beta}\left(\vec{v}^{\omega}\right):=-\partial_{\alpha \beta}^{2} v_{3}^{\omega}=-\frac{\partial^{2} v_{3}^{w}}{\partial x_{a} \partial x_{\beta}} \\
& M_{\alpha \beta}\left(\vec{v}^{\omega}\right):=\frac{2 E_{\omega} t^{3}}{3\left(1-\nu_{\omega}^{2}\right)}\left\{\left(1-\nu_{\omega}\right) K_{\alpha \beta}\left(\vec{v}^{\omega}\right)+\nu_{\omega} K_{\iota \iota}\left(\vec{v}^{\omega}\right) \delta_{a \beta}\right\}, \alpha, \beta=1,2 .
\end{aligned}
$$

Here $E_{\Omega}>0$ and $E_{\omega}>0$ denote Young's modulus of the body $\Omega$ and the plate $\omega^{t}$ (denoted also by $\omega$ in what follows), while, $\nu_{\Omega}$ and $\nu_{\omega} \in(0,1)$ represent the corresponding Poisson ratios. Throughout this paper, Latin indices have values $\{1,2,3\}$, Greek indices take the values $\{1,2\}$, respectively. We also use the summation convention whereby summation is implied when an index is repeated exactly two times. As usual, the constant $C$ (with or without a subscript) denotes a generic constant independent of related parameters (especially the finite element mesh size $h$ ), which may take different values in different places.

With this notation, the mathematical model of the body-plate problem is to find $\vec{u}:=\left(\vec{u}^{\Omega}, \vec{u}^{\omega}\right) \in V$ such that

$$
J(\vec{u})=\min _{\vec{v} \in V} J(\vec{v}),
$$

or equivalently, find $\vec{u}:=\left(\vec{u}^{\Omega}, \vec{u}^{\omega}\right) \in V$ such that

$$
D(\vec{u}, \vec{v})=F(\vec{v}), \quad \forall \vec{v} \in V,
$$

where

$$
\begin{array}{ll}
V:=\left\{\vec{v}=\left(\vec{v}^{\Omega}, \vec{v}^{\omega}\right):\right. & \vec{v}^{\Omega} \in\left(H^{1}(\Omega)\right)^{3}, \vec{v}^{\omega} \in\left(H^{1}(\omega)\right)^{2} \times H^{2}(\omega), \vec{v}^{\Omega}=\vec{v}^{\omega} \text { on } \omega_{b}, \\
& \left.v_{\alpha}^{\omega}=0, \alpha=1,2, \text { on } \gamma_{0}, v_{3}^{\omega}=\partial_{2} v_{3}^{\omega}=0 \text { on } \gamma_{0}\right\} .
\end{array}
$$

Throughout this paper we adopt the standard definitions of Sobolev spaces (cf. $[11,20]$ ) and the standard conventions for Sobolev norms and seminorms of a function $v$ defined on an open set $G$ :

$$
\|v\|_{m, G}:=\left(\int_{G} \sum_{|\alpha| \leq m}\left|\partial^{\alpha} v\right|^{2} d x\right)^{1 / 2}, \quad|v|_{m, G}:=\left(\int_{G} \sum_{|\alpha|=m}\left|\partial^{\alpha} v\right|^{2} d x\right)^{1 / 2} .
$$

Meanwhile, for a vector-valued function $\vec{v}=\left(v_{1}, v_{2}, \cdots, v_{l}\right) \in\left(H^{s}(G)\right)^{l}$,

$$
\|\vec{v}\|_{s, G}:=\left(\sum_{i=1}{ }^{l}\left\|v_{i}\right\|_{s, G}^{2}\right)^{1 / 2}, \quad|\vec{v}|_{s, G}:=\left(\sum_{i=1}{ }^{l}\left|v_{i}\right|_{s, G}^{2}\right)^{1 / 2}
$$

etc. In what follows, we also denote by $P_{l}(G)$ the space of polynomials of total degree no more than $l$ on $G$, and by $Q_{l}(G)$ the space of polynomials of degree no more than $l$ with respect to each variable $x_{i}$ on $G$.

The unique solvability of (2.6) was proved in [24] by the Lax-Milgram lemma. By Green's formula for partial integration, we can also obtain the partial differential system of the body-plate problem (2.6) (cf. [24]). For simplicity, we list here only the induced "force" junctions on the interface $\omega_{b}$, which will be used later on:

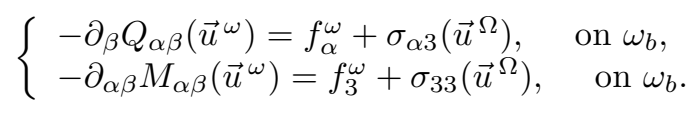


The equalities (2.7) can be explained as follows. Due to the rigid contact of the body $\Omega$ with the plate $\omega$, the body $\Omega$ gives an extra applied surface force to the plate $\omega$ on the interface part $\omega_{b}$.

We proceed to present the finite element approximation of (2.6). Let $\bar{\omega}:=$ $\bigcup_{\tau \in T_{h}^{\omega}} \bar{\tau}$ be the quasi-uniform rectangular subdivision of $\omega$, with each element $\tau$ being an open rectangle of size $h$. By quasi-uniformity we mean that there exist two positive constants $C_{0}$ and $C_{1}$, independent of $h$, such that each rectangle $\tau \in T_{h}^{\omega}$ contains (resp. is contained in) a disk of radius $C_{0} h$ (resp. $\left.C_{1} h\right)$. We also assume that the boundary of $\omega_{b}$ is aligned with the subdivision $T_{h}^{\omega}$ (cf. [13]), which means that there exists no rectangle $\tau \in T_{h}^{\omega}$ which is cut into two parts by the boundary of $\omega_{b}$. Similarly, let $\bar{\Omega}:=\bigcup_{K \in T_{h}^{\Omega}} \bar{K}$ denote the quasi-uniform cuboidal subdivision of $\Omega$ such that each element $\tau \subset \omega_{b}$ is the boundary surface of a certain finite element $K \in T_{h}^{\Omega}$. For simplicity, we also denote by $h$ the mesh size of the subdivision $T_{h}^{\Omega}$.

We denote by $V_{h}^{1}(\omega)$ (resp. $V_{h}^{1}(\Omega)$ ) the space of all piecewise bilinear (resp. trilinear) continuous functions associated with the subdivision $T_{h}^{\omega}\left(\operatorname{resp} . T_{h}^{\Omega}\right.$ ). We denote by $V_{h}^{2}(\omega)$ the usual Adini finite element space associated with the subdivision $T_{h}^{\omega}$ (cf. [11, p. 364]); that is, for each $\tau \in T_{h}^{\omega}$, with $\left\{a_{i}\right\}_{i=1}^{4}$ as its four vertices respectively, the shape function space consists of all polynomials of the form

$$
P: x=\left(x_{1}, x_{2}\right) \rightarrow p(x)=\sum_{\alpha_{1}+\alpha_{2} \leq 3} b_{\alpha_{1} \alpha_{2}} x_{1}^{\alpha_{1}} x_{2}^{\alpha_{2}}+b_{13} x_{1} x_{2}^{3}+b_{31} x_{1}^{3} x_{2},
$$

which are uniquely determined by the degrees of freedom

$$
\Sigma_{\tau}=\left\{p\left(a_{i}\right), \partial_{1} p\left(a_{i}\right), \partial_{2} p\left(a_{i}\right), 1 \leq i \leq 4\right\} .
$$

We now set

$$
\begin{aligned}
V_{h}:=\left\{\vec{v}=\left(\vec{v}^{\Omega}, \vec{v}^{\omega}\right): \vec{v}_{h}^{\Omega} \in\left(V_{h}^{1}(\Omega)\right)^{3}, v_{\alpha, h}^{\omega} \in V_{\omega}^{1}(\omega), \alpha=1,2, v_{3, h}^{\omega} \in V_{h}^{2}(\omega),\right. \\
\\
\vec{v}_{h}^{\omega}(Q)=\partial_{1} v_{3, h}^{\omega}(Q)=\partial_{2} v_{3, h}^{\omega}(Q)=0, \forall \operatorname{nodes} Q \in \gamma_{0}, \\
\text { and } \left.\vec{v}_{h}^{\Omega}(Q)=\vec{v}_{h}^{\omega}(Q), \forall \operatorname{nodes} Q \in \omega_{b}\right\},
\end{aligned}
$$

and for $\vec{v}_{h}, \vec{w}_{h} \in V_{h}$ we define

$$
D_{h}\left(\vec{v}_{h}, \vec{w}_{h}\right):=D_{\Omega, h}\left(\vec{v}_{h}^{\Omega}, \vec{w}_{h}^{\Omega}\right)+D_{\omega, h}\left(\vec{v}_{h}^{\omega}, \vec{w}_{h}^{\omega}\right),
$$

where

$$
\begin{aligned}
& D_{\Omega, h}\left(\vec{v}_{h}^{\Omega}, \vec{w}_{h}^{\Omega}\right):=\int_{\Omega} \sigma_{i j}\left(\vec{v}_{h}^{\Omega}\right) \varepsilon_{i j}\left(\vec{w}_{h}^{\Omega}\right) d \Omega \\
& D_{\omega, h}\left(\vec{v}_{h}^{\omega}, \vec{w}_{h}^{\omega}\right):= \int_{\omega} Q_{\alpha \beta}\left(\vec{v}_{h}^{\omega}\right) \varepsilon_{\alpha \beta}\left(\vec{w}_{h}^{\omega}\right) d \omega \\
&+\sum_{\tau \in T_{h}^{\omega}} \int_{\tau} M_{\alpha \beta}\left(\vec{v}_{h}^{\omega}\right) K_{\alpha \beta}\left(\vec{w}_{h}^{\omega}\right) d \tau .
\end{aligned}
$$

Then the finite element approximation of (2.6) can be expressed by finding $\vec{u}_{h}=$ $\left(\vec{u}_{h}^{\Omega}, \vec{u}_{h}^{\omega}\right) \in V_{h}$ such that

$$
D_{h}\left(\vec{u}_{h}, \vec{v}_{h}\right)=F\left(\vec{v}_{h}\right), \quad \forall \vec{v}_{h} \in V_{h} .
$$

In practice, other high order finite elements are acceptable choices to approximate problem (2.6). For simplicity of presentation, we consider here only the discretization method proposed in [24]. 


\section{Some BASIC RESUlts}

In this section, let us give some basic results that will be needed later. We first define

$$
V_{h}^{1}\left(\Omega ; \omega_{b}\right):=\left\{v \in V_{h}^{1}(\Omega): v(Q)=0, \forall \text { nodes } Q \in \omega_{b}\right\} .
$$

To simplify the presentation hereafter, we also define

$$
\|v\|_{k, h, G}:=\left(\sum_{\tau \in T_{h}^{\omega} \cap G}\|v\|_{k, \tau}{ }^{2}\right)^{1 / 2}, \quad|v|_{k, h, G}:=\left(\sum_{\tau \in T_{h}^{\omega} \cap G}|v|_{k, \tau}^{2}\right)^{1 / 2},
$$

where $G$ is any open subset of $\omega$ aligned with the finite element subdivision $T_{h}^{\omega}$, and $v$ is smooth on each rectangular element $\tau \in T_{h}^{\omega}$.

Lemma 1. There exists a positive constant $C$, independent of the mesh size $h$, such that for any vector-valued function $\vec{v} \in\left(V_{h}^{1}(\Omega)\right)^{3}$ satisfying

$$
D_{\Omega, h}(\vec{v}, \vec{w})=0, \quad \forall \vec{w} \in\left(V_{h}^{1}\left(\Omega ; \omega_{b}\right)\right)^{3},
$$

it admits the following estimate:

$$
D_{\Omega, h}(\vec{v}, \vec{v}) \leq C\|\vec{v}\|_{\frac{1}{2}, \omega_{b}}^{2}
$$

where $\|\cdot\|_{\frac{1}{2}, \omega_{b}}$ denotes the standard fractional order Sobolev norm (cf. [17] [20]), i.e.,

$$
\|v\|_{\frac{1}{2}, \omega_{b}}^{2}:=\|v\|_{0, \omega_{b}}^{2}+\int_{\omega_{b}} \int_{\omega_{b}} \frac{\left|v\left(\bar{x}_{0}\right)-v\left(\bar{y}_{0}\right)\right|^{2}}{\left|\bar{x}_{0}-\bar{y}_{0}\right|^{3}} d x_{1} d x_{2} d y_{1} d y_{2}
$$

where $\bar{x}_{0}=\left(x_{1}, x_{2}, 0\right)$ and $\bar{y}_{0}=\left(y_{1}, y_{2}, 0\right)$.

Proof. The proof is standard to some extent (cf. [3, 5]). We first introduce an auxiliary problem: Find $\vec{u} \in\left(H^{1}(\Omega)\right)^{3}$ with $\vec{u}=\vec{v}$ on $\omega_{b}$ such that

$$
D_{\Omega, h}(\vec{u}, \vec{w})=0, \quad \forall \vec{w} \in\left(H^{1}(\Omega)\right)^{3} \text { with } \vec{w}=0 \text { on } \omega_{b} .
$$

For the cuboidal domain $\Omega$ considered here, there always exists some $\varepsilon \in\left(0, \frac{1}{2}\right)$ such that $\vec{u}$ is in $\left(H^{1+\varepsilon}(\Omega)\right)^{3}$ and admits the regularity estimates (cf. [17, 20])

$$
\|\vec{u}\|_{1+\varepsilon, \Omega} \leq C\|\vec{v}\|_{\frac{1}{2}+\varepsilon, \omega_{b}}, \quad\|\vec{u}\|_{1, \Omega} \leq C\|\vec{v}\|_{\frac{1}{2}, \omega_{b}} .
$$

Let $I_{h}^{\Omega}$ denote the usual piecewise trilinear interpolation operator associated with the finite element space $V_{h}^{1}(\Omega)$. Then, noting that $\vec{v}$ is just the finite element approximate solution of (3.2), by Cea's lemma we easily have (cf. [11, p. 104])

$$
D_{\Omega, h}(\vec{v}, \vec{v}) \leq C\left[\|\vec{u}-\vec{v}\|_{1, \Omega}^{2}+\|\vec{u}\|_{1, \Omega}^{2}\right] \leq C\left[\left\|\vec{u}-I_{h}^{\Omega} \vec{u}\right\|_{1, \Omega}^{2}+\|\vec{u}\|_{1, \Omega}^{2}\right]
$$

which, together with (3.3), the interpolation estimate of $I_{h}^{\Omega}$ and the inverse inequality (cf. [11, p.122], [1]), implies

$$
\begin{aligned}
D_{\Omega, h}(\vec{v}, \vec{v}) & \leq C\left[h^{2 \varepsilon}\|\vec{u}\|_{1+\varepsilon, \Omega}^{2}+\|\vec{v}\|_{\frac{1}{2}, \omega_{b}}^{2}\right] \leq C\left[h^{2 \varepsilon}\|\vec{u}\|_{\frac{1}{2}+\varepsilon, \omega_{b}}^{2}+\|\vec{v}\|_{\frac{1}{2}, \omega_{b}}^{2}\right] \\
& \leq C\|\vec{v}\|_{\frac{1}{2}, \omega_{b}}^{2} .
\end{aligned}
$$

The desired result then follows.

To obtain some estimates related to functions in the Adini finite element space, we now introduce a transfer operator $E_{h}$ which builds an important bridge between $V_{h}^{2}(\omega)$ and its conforming relative: the Bogner-Fox-Schmit bi-cubic element (cf. [7, 8, 9]). The definition is as follows. Let $\tau$ be a rectangle in $T_{h}^{\omega}$, with $p_{i}, 1 \leq i \leq 4$, 
as its four vertices. Then $\left.\left(E_{h} v\right)\right|_{\tau} \in Q_{3}(\tau)$ for any $v \in V_{h}^{2}(\omega)$, and it satisfies the interpolation conditions

$$
\left\{\begin{array}{l}
\left(E_{h} v\right)\left(p_{i}\right)=v\left(p_{i}\right), \quad 1 \leq i \leq 4 \\
{\left[\partial_{\alpha}\left(E_{h} v\right)\right]\left(p_{i}\right)=\left(\partial_{\alpha} v\right)\left(p_{i}\right), \quad 1 \leq i \leq 4, \alpha=1,2} \\
{\left[\partial_{12}\left(E_{h} v\right)\right]\left(p_{i}\right)=0, \quad 1 \leq i \leq 4}
\end{array}\right.
$$

For this transfer operator, we have the estimate (cf. [7, 8, 9])

$$
\sum_{i=0}^{2} h^{i}\left|v-E_{h} v\right|_{i, \tau} \leq C h^{2}|v|_{2, \tau}, \quad \forall \tau \in T_{h}^{\omega}, \forall v \in V_{h}^{2}(\omega) .
$$

Lemma 2. There exists a positive constant $C$, independent of $h$, such that, for any $v \in V_{h}^{2}(\omega)$ with zero nodal parameters on $\gamma_{0}$ (i.e., $v(Q)=\partial_{1} v(Q)=\partial_{2} v(Q)=0$, $\forall$ nodes $\left.Q \in \gamma_{0}\right)$, we have

$$
\|v\|_{2, h, \omega} \leq C|v|_{2, h, \omega} .
$$

This is just the so-called Poincaré type inequality for the Adini finite element space.

Proof. The result can be verified via the techniques in 23. But it seems more convenient to obtain this result with the help of the interpolation operator $E_{h}$. In this way, we can also show more clearly that the generic constant $C$ is independent of the finite element mesh size $h$. It is easy to see by a compactness argument (cf. [26]) that there exists a positive constant $C$ such that, for any $w \in H^{2}(\omega)$ with $w=\partial_{2} w=0$ on $\gamma_{0}$,

$$
\|w\|_{2, \omega} \leq C|w|_{2, \omega}
$$

which is just the conventional Poincaré type inequality.

Note that $E_{h} v \in H^{2}(\omega)$ and $E_{h} v=\partial_{2}\left(E_{h} v\right)=0$ on $\gamma_{0}$. Thus it follows from the estimates (3.6) and (3.8) that

$$
\begin{aligned}
\|v\|_{2, h, \omega} & \leq\left\|v-E_{h} v\right\|_{2, h, \omega}+\left\|E_{h} v\right\|_{2, \omega} \leq C\left[|v|_{2, h, \omega}+\left|E_{h} v\right|_{2, \omega}\right] \\
& \leq C|v|_{2, h, \omega} .
\end{aligned}
$$

The desired result then follows.

We also define

$$
\begin{gathered}
V_{h}^{1}\left(\omega ; \gamma_{0}\right):=\left\{v \in V_{h}^{1}(\omega): v(Q)=0, \forall \text { nodes } Q \in \gamma_{0}\right\}, \\
V_{h}^{2}\left(\omega ; \gamma_{0}\right):=\left\{v \in V_{h}^{2}(\omega): v(Q)=\partial_{1} v(Q)=\partial_{2} v(Q)=0, \forall \text { nodes } Q \in \gamma_{0}\right\},
\end{gathered}
$$
and $V_{h}^{2}(G):=\left.V_{h}^{2}(\omega)\right|_{G}, V_{h}^{1}(G):=\left.V_{h}^{1}(\omega)\right|_{G}$, where $G$ is any subset in $\omega$ with the boundary $\partial G$ aligned with the subdivision $T_{h}^{\omega}$.

Any vector-valued function $\vec{v}^{\omega} \in\left(V_{h}^{1}\left(\omega ; \gamma_{0}\right)\right)^{2} \times V_{h}^{2}\left(\omega ; \gamma_{0}\right)$ can be associated with a unique vector-valued function $\left[\vec{v}^{\omega}\right]^{\Omega} \in\left(V_{h}^{1}(\Omega)\right)^{3}$ such that

$$
\left\{\begin{array}{l}
{\left[\vec{v}^{\omega}\right]^{\Omega}(Q)=\vec{v}(Q), \quad \forall \text { nodes } Q \in \omega_{b}} \\
D_{\Omega, h}\left(\left[\vec{v}^{\omega}\right]^{\Omega}, \vec{w}\right)=0, \quad \forall \vec{w} \in\left(V_{h}^{1}\left(\Omega ; \omega_{b}\right)\right)^{3} .
\end{array}\right.
$$

Any function $v \in V_{h}^{2}\left(\omega_{b}\right)$ can be associated with a unique extension function $\hat{v} \in$ $V_{h}^{2}\left(\omega ; \gamma_{0}\right)$ such that

$$
\left\{\begin{array}{l}
\hat{v}(Q)=v(Q), \partial_{\alpha} \hat{v}(Q)=\partial_{\alpha} v(Q), \alpha=1,2, \forall \text { nodes } Q \in \gamma_{1}:=\partial \omega_{b} \cap \partial \omega_{c}, \\
\sum_{\tau \in T_{h}^{\omega} \cap \omega_{c}} \int_{\tau} M_{\alpha \beta}(\hat{v}) K_{\alpha \beta}(w) d \tau=0
\end{array}\right.
$$


where $\omega_{c}:=\omega \backslash \omega_{b}$, and $w \in V_{h}^{2}\left(\omega_{c}\right)$ with $w(Q)=\partial_{\alpha} w(Q)=0, \alpha=1,2, \forall$ nodes $Q \in \gamma_{0}$ or $\gamma_{1}$. Note that only the third component of a vector-valued function $\vec{v}$ is used in the definitions of $M_{\alpha \beta}(\vec{v})$ and $K_{\alpha \beta}(\vec{v})$ (cf. (2.5)). Thus, for a scalar function $v$, we naturally define $M_{\alpha \beta}(v)$, and $K_{\alpha \beta}(v)$ by $(2.5)$ in such a way of viewing the scalar function $v$ as the third component of a certain vector-valued function $\vec{v}$. We will use the similar conventions for other definitions given in Section 2.

Lemma 3. There exist two positive constants $C_{1}$ and $C_{2}$, independent of $h$, such that, for any function $v \in V_{h}^{2}\left(\omega_{b}\right)$,

$$
C_{1}\|v\|_{2, h, \omega_{b}}^{2} \leq \sum_{\tau \in T_{h}^{\omega}} \int_{\tau} M_{\alpha \beta}(\hat{v}) K_{\alpha \beta}(\hat{v}) d \tau \leq C_{2}\|v\|_{2, h, \omega_{b}}^{2} .
$$

Proof. It is clear that (cf. [7])

$$
A\left(1-\nu_{\omega}\right)|\hat{v}|_{2, h, \omega}^{2} \leq \sum_{\tau \in T_{h}^{\omega}} \int_{\tau} M_{\alpha \beta}(\hat{v}) K_{\alpha \beta}(\hat{v}) d \tau \leq A\left(1-\nu_{\omega}\right)|\hat{v}|_{2, h, \omega}^{2},
$$

where $A:=\frac{2 E_{\omega} t}{1-\nu_{\omega}^{2}}$ stands for the rigid coefficient of the plate $\omega$.

Therefore it suffices to verify the following result, which implies (3.12):

$$
C_{1}\|v\|_{2, h, \omega_{b}} \leq|\hat{v}|_{2, h, \omega} \leq C_{2}\|v\|_{2, h, \omega_{b}} .
$$

The left-hand inequality of 3.13 follows at once from Lemma 2 . In order to prove the right-hand inequality of (3.13), we first introduce an auxiliary problem:

$$
\left\{\begin{array}{l}
u \in H^{2}\left(\omega_{c}\right), \quad u=E_{h} \hat{v}, \quad \partial_{2} u=\partial_{2}\left(E_{h} \hat{v}\right), \quad \text { on } \gamma_{0} \text { and } \gamma_{1}, \\
\int_{\omega_{c}} M_{\alpha \beta}(u) K_{\alpha \beta}(w) d \omega=0, \quad \forall w \in H^{2}\left(\omega_{c}\right) \text { with } w=\partial_{2} w=0 \text { on } \gamma_{0} \cup \gamma_{1},
\end{array}\right.
$$

where $E_{h}$ is defined by (3.5).

Then for the domain $\omega_{c}$ with shape given in this paper, there always exists some $\varepsilon \in\left(0, \frac{1}{2}\right)$ such that $u$ is in $H^{2+\varepsilon}\left(\omega_{c}\right)$ and admits the regularity estimates (cf. [4, 17])

$$
\left\{\begin{array}{l}
\|u\|_{2+\varepsilon, \omega_{c}} \leq C\left[\|u\|_{\frac{3}{2}+\varepsilon, \gamma_{1}}+\left\|\partial_{2} u\right\|_{\frac{1}{2}, \gamma_{1}}\right] \\
\|u\|_{2, \omega_{c}} \leq C\left[\|u\|_{\frac{3}{2}, \gamma_{1}}+\left\|\partial_{2} u\right\|_{\frac{1}{2}, \gamma_{1}}\right]
\end{array}\right.
$$

Note that $\hat{v}$ (the restriction of $\hat{v}$ to $\omega_{c}$, still denoted by $\hat{v}$ for simplicity) is just the approximate solution of the continuous problem (3.14) by the Adini finite element method. We then have (cf. [11])

$$
\|u-\hat{v}\|_{2, h, \omega_{c}} \leq C h^{\varepsilon}\|u\|_{2+\varepsilon, \omega_{c}} .
$$

Hence, by the inverse inequality, 3.15 and (3.16) we know that

$$
\begin{aligned}
|\hat{v}|_{2, h, \omega_{c}} & \leq|u-\hat{v}|_{2, h, \omega_{c}}+|u|_{2, h, \omega_{c}} \\
& \leq C\left[h^{\varepsilon}\left(\|u\|_{\frac{3}{2}+\varepsilon, \gamma_{1}}+\left\|\partial_{2} u\right\|_{\frac{1}{2}, \gamma_{1}}\right)+\|u\|_{\frac{3}{2}, \gamma_{1}}+\left\|\partial_{2} u\right\|_{\frac{1}{2}, \gamma_{1}}\right] \\
& \leq C\left[\|u\|_{\frac{3}{2}, \gamma_{1}}+\left\|\partial_{2} u\right\|_{\frac{1}{2}, \gamma_{1}}\right]=C\left[\left\|E_{h} \hat{v}\right\|_{\frac{3}{2}, \gamma_{1}}+\left\|\partial_{2}\left(E_{h} \hat{v}\right)\right\|_{\frac{1}{2}, \gamma_{1}}\right] .
\end{aligned}
$$

On the other hand, it follows from the trace theorem (cf. [17, 20]) and the estimate (3.6) that

$$
\left\|E_{h} \hat{v}\right\|_{\frac{3}{2}, \gamma_{1}}+\left\|\partial_{2}\left(E_{h} \hat{v}\right)\right\|_{\frac{1}{2}, \gamma_{1}} \leq C\left\|E_{h} \hat{v}\right\|_{2, \omega_{b}} \leq C\|\hat{v}\|_{2, \omega_{b}}=C\|v\|_{2, \omega_{b}} .
$$

The right-hand inequality of (3.13) then follows from (3.17) and (3.18) directly. 
It should be pointed out that even for $v \in V_{h}^{2}\left(\omega ; \gamma_{0}\right)$, we also have the following result, similar to the left-hand inequality of (3.11):

$$
\|v\|_{2, h, \omega_{b}}^{2} \leq C \sum_{\tau \in T_{h}^{\omega}} \int_{\tau} M_{\alpha \beta}(v) K_{\alpha \beta}(v) d \tau .
$$

Lemma 4. There exists a positive constant $C^{*}$, independent of $h$, such that for any vector-valued function $\vec{v}^{\omega} \in\left(V_{h}^{1}\left(\omega ; \gamma_{0}\right)\right)^{2} \times V_{h}^{2}\left(\omega ; \gamma_{0}\right)$ we have

$$
D_{\Omega, h}\left(\left[\vec{v}^{\omega}\right]^{\Omega},\left[\vec{v}^{\omega}\right]^{\Omega}\right) \leq C^{*} D_{\omega, h}\left(\vec{v}^{\omega}, \vec{v}^{\omega}\right),
$$

where $\left[\vec{v}^{\omega}\right]^{\Omega}$ is defined by (3.9).

Proof. Define $\vec{w}:=\left.\left[\vec{v}^{\omega}\right]^{\Omega}\right|_{\omega_{b}}$. It follows from Lemma 1 that

$$
D_{\Omega, h}\left(\left[\vec{v}^{\omega}\right]^{\Omega},\left[\vec{v}^{\omega}\right]^{\Omega}\right) \leq C\|\vec{w}\|_{\frac{1}{2}, \omega_{b}}^{2}
$$

By the trace theorem and the Korn inequality (cf. [20]) we have $\left(\vec{w}:=\left(w_{i}\right)\right)$

$$
\begin{aligned}
\left\|w_{1}\right\|_{\frac{1}{2}, \omega_{b}}^{2}+\left\|w_{2}\right\|_{\frac{1}{2}, \omega_{b}}^{2} & \leq C\left[\left\|v_{1}^{\omega}\right\|_{1, \omega}^{2}+\left\|v_{2}^{\omega}\right\|_{1, \omega}^{2}\right] \\
& \leq C \int_{\omega} Q_{\alpha \beta}\left(\vec{v}^{\omega}\right) \varepsilon_{\alpha \beta}\left(\vec{v}^{\omega}\right) d \omega .
\end{aligned}
$$

Moreover, by the interpolation estimate of $I_{h}^{\Omega}$, the trace theorem, the embedding inequality and (3.19) we get

$$
\left\|w_{3}\right\|_{\frac{1}{2}, \omega_{b}}^{2}=\left\|I_{h}^{\Omega} v_{3}^{\omega}\right\|_{\frac{1}{2}, \omega_{b}}^{2} \leq C\left\|v_{3}^{\omega}\right\|_{2, h, \omega_{b}}^{2} \leq C \sum_{\tau \in T_{h}^{\omega}} \int_{\tau} M_{\alpha \beta}\left(v_{3}^{\omega}\right) K_{\alpha \beta}\left(v_{3}^{\omega}\right) d \tau .
$$

The desired result follows from (3.21), (3.22) and (3.23).

Lemma 5. For any vector-valued functions $\vec{v}^{\Omega} \in\left(V_{h}^{1}(\Omega)\right)^{3}$ and $\vec{v}^{\omega} \in\left(V_{h}^{1}\left(\omega ; \gamma_{0}\right)\right)^{2} \times$ $V_{h}^{2}\left(\omega ; \gamma_{0}\right)$ which satisfy

$$
\left\{\begin{array}{l}
D_{\Omega, h}\left(\vec{v}^{\Omega}, \vec{w}\right)=0, \quad \forall \vec{w} \in\left(V_{h}^{1}\left(\Omega ; \omega_{b}\right)\right)^{3} \\
D_{\Omega, h}\left(\vec{v}^{\Omega},[\vec{w}]^{\Omega}\right)+D_{\omega, h}\left(\vec{v}^{\omega}, \vec{w}\right)=0, \quad \forall \vec{w} \in\left(V_{h}^{1}\left(\omega ; \gamma_{0}\right)\right)^{2} \times V_{h}^{2}\left(\omega ; \gamma_{0}\right),
\end{array}\right.
$$

we have the estimate

$$
D_{\omega, h}\left(\vec{v}^{\omega}, \vec{v}^{\omega}\right) \leq C^{*} D_{\Omega, h}\left(\vec{v}^{\Omega}, \vec{v}^{\Omega}\right),
$$

where the generic constant $C^{*}$ is the same as in Lemma 4 .

Proof. Taking $\vec{w}=\vec{v}^{\omega}$ in the second equation of (3.24), we find from Lemma4 and the Cauchy-Schwarz inequality that

$$
\begin{aligned}
D_{\omega, h}\left(\vec{v}^{\omega}, \vec{v}^{\omega}\right) & =-D_{\Omega, h}\left(\vec{v}^{\Omega},\left[\vec{v}^{\omega}\right]^{\Omega}\right) \\
& \leq D_{\Omega, h}^{1 / 2}\left(\vec{v}^{\Omega}, \vec{v}^{\Omega}\right) D_{\Omega, h}^{1 / 2}\left(\left[\vec{v}^{\omega}\right]^{\Omega},\left[\vec{v}^{\omega}\right]^{\Omega}\right) \\
& \leq \sqrt{C^{*}} D_{\Omega, h}^{1 / 2}\left(\vec{v}^{\Omega}, \vec{v}^{\Omega}\right) D_{\omega, h}^{1 / 2}\left(\vec{v}^{\omega}, \vec{v}^{\omega}\right),
\end{aligned}
$$

which leads to the inequality (3.25)). 


\section{Nonoverlapping DOMAin DECOMPOSition Methods AND CONDITION NUMBER ESTIMATES}

In this section, we give some numerical solvers for the problem (2.8), which yields a large sparse symmetric positive definite linear algebraic system

$$
A \bar{u}=\bar{f}
$$

when describing it in matrix form according to the nodal parameters.

Considering the specialty of such a problem, the nonoverlapping domain decomposition method is a natural and reasonable choice (cf. [12]). Here let us first introduce a nonoverlapping domain decomposition method for solving (2.8) (or equivalently, (4.1)) via the basic strategy given in [3] 5, 18. To this end, we list the nodal parameters $\bar{u}$ in proper order such that the index set $I=I_{1} \cup I_{2}$, where $I_{1}$ consists of the indices of nodal parameters with nodes belonging to $\bar{\Omega} \backslash \bar{\omega}_{b}$, whereas $I_{2}$ consists of the indices of nodal parameters related to the remaining nodes. Then the linear system (4.1) takes the block form

$$
\left[\begin{array}{ll}
A_{11} & A_{12} \\
A_{12}^{t} & A_{22}
\end{array}\right]\left[\begin{array}{l}
\bar{u}_{1} \\
\bar{u}_{2}
\end{array}\right]=\left[\begin{array}{c}
\bar{f}_{1} \\
\bar{f}_{2}
\end{array}\right]
$$

By the block Gaussian elimination, problem (4.2) reduces to an equivalent system of the unknowns $\bar{u}_{2}$ given by

$$
S \bar{u}_{2}=\tilde{f}_{2},
$$

where $S:=A_{22}-A_{12}^{t} A_{11}^{-1} A_{12}, \tilde{f}:=\bar{f}_{2}-A_{12}^{t} A_{11}^{-1} \bar{f}_{1}$. The matrix $S$ is referred to as the Schur complement $A_{22}$ in $A$. According to the construction of $I_{2}$, it is clear that all the components of $\bar{u}_{2}$ are the nodal parameters related to the plate problem.

If we obtain $\bar{u}_{2}$ by solving (4.3), we can then get $\bar{u}_{1}$ by solving the subsystem

$$
A_{11} \bar{u}_{1}=\bar{f}_{1}-A_{12} \bar{u}_{2} .
$$

We point out that the solution of the subsystem with the typical form $A_{11} \bar{u}_{1}=\bar{g}_{1}$ amounts to solving a body problem with prescribed displacements on $\omega_{b}$ and with the free boundary conditions on $\partial \Omega \backslash \omega_{b}$, i.e.,

$$
\left\{\begin{array}{l}
\vec{v} \in\left(V_{h}^{1}(\Omega)\right)^{3}, \\
D_{\Omega, h}(\vec{v}, \vec{w})=g_{1}(\vec{w}), \quad \forall \vec{w} \in\left(V_{h}^{1}\left(\Omega ; \omega_{b}\right)\right)^{3} .
\end{array}\right.
$$

Thus we can utilize known effective algorithms to carry out this step.

Since the Schur complement $S$ is dense and ill-conditioned, it is common practice to solve the Schur complement system (4.3) iteratively via preconditioned conjugated gradient methods. To enhance the efficiency of computation, we need to propose an effective preconditioner associated with the Schur complement $S$. Fortunately, the following theorem states that the symmetric positive definite matrix $\bar{S}$ related to the bilinear form $D_{\omega, h}(\cdot, \cdot)$ is an optimal one. To see this, we first give the detailed description of $\bar{S}$. Let $[\because \cdot \cdot$ denote the standard euclidean inner product. For any $\vec{v}^{\omega}, \vec{w}^{\omega} \in\left(V_{h}^{1}\left(\omega ; \gamma_{0}\right)\right)^{2} \times V_{h}^{2}\left(\omega ; \gamma_{0}\right)$, we denote by $\bar{v}_{2}$ and $\bar{w}_{2}$ their nodal parameters respectively, which inherit the same order determined by the index set $I_{2}$. Then $\bar{S}$ is just the symmetric positive definite matrix given by

$$
\left[\bar{S} \bar{v}_{2}, \bar{w}_{2}\right]=D_{\omega, h}\left(\vec{v}^{\omega}, \vec{w}^{\omega}\right) .
$$


Theorem 1. There exist two positive constants $C_{1}$ and $C_{2}$, which are independent of the mesh size $h$, such that

$$
C_{1} \leq \operatorname{Cond}_{2}\left(\bar{S}^{-1} S\right):=\lambda_{\max }\left(\bar{S}^{-1} S\right) / \lambda_{\min }\left(\bar{S}^{-1} S\right) \leq C_{2} .
$$

Proof. With the help of Lemma 3 or (3.19), the proof is formal (cf. [3, 5]). By the Rayleigh representation theorem, it suffices to show that for any nonzero nodal parameter vector $\bar{v}_{2}$ the following estimate holds:

$$
C_{1}\left[\bar{S} \bar{v}_{2}, \bar{v}_{2}\right] \leq\left[S \bar{v}_{2}, \bar{v}_{2}\right] \leq C_{2}\left[\bar{S} \bar{v}_{2}, \bar{v}_{2}\right] .
$$

Let $\vec{v}^{\omega} \in\left(V_{h}^{1}\left(\omega ; \gamma_{0}\right)\right)^{2} \times V_{h}^{2}\left(\omega ; \gamma_{0}\right)$ denote the related vector-valued function with $\bar{v}_{2}$ as its nodal parameters. Then from the definition (4.6) we have

$$
\left[\bar{S} \bar{v}_{2}, \bar{v}_{2}\right]=D_{\omega, h}\left(\vec{v}^{\omega}, \vec{v}^{\omega}\right) \text {. }
$$

On the other hand, let $\left[\vec{v}^{\omega}\right]^{\Omega} \in\left(V_{h}^{1}(\Omega)\right)^{3}$ be the related vector-valued function defined by (3.9). It is easy to check that (cf. [3, [5])

$$
\left[S \bar{v}_{2}, \bar{v}_{2}\right]=D_{\Omega, h}\left(\left[\vec{v}^{\omega}\right]^{\Omega},\left[\vec{v}^{\omega}\right]^{\Omega}\right)+D_{\omega, h}\left(\vec{v}^{\omega}, \vec{v}^{\omega}\right) .
$$

(4.8) follows immediately from (3.20), (4.9) and (4.10).

Note also that the solution of the problem $\bar{S} \bar{u}_{2}=\bar{g}_{2}$ amounts to solving the related elastic plate problem, i.e.,

$$
\left\{\begin{array}{l}
\vec{v}^{\omega} \in\left(V_{h}^{1}\left(\omega ; \gamma_{0}\right)\right)^{2} \times V_{h}^{2}\left(\omega ; \gamma_{0}\right), \\
D_{\omega, h}\left(\vec{v}^{\omega}, \vec{w}^{\omega}\right)=g_{2}\left(\vec{w}^{\omega}\right), \quad \forall \vec{w}^{\omega} \in\left(V_{h}^{1}\left(\omega ; \gamma_{0}\right)\right)^{2} \times V_{h}^{2}\left(\omega ; \gamma_{0}\right)
\end{array}\right.
$$

which can be solved by existing efficient solvers.

Now we proceed to give another nonoverlapping domain decomposition method for solving (2.8). The main ideas arise in some sense from [19, 21]. We first consider the continuous version of this method. We know that once the displacements on $\omega_{b}$ are obtained, the global problem (2.8) can then be transformed into two separate subproblems - the elastic body problem and the elastic plate problem. Based on this observation and noting the induced "force" junctions on the interface $\omega_{b}$ (cf. (2.7)), we can design an iterative algorithm for solving (2.8). That is, given arbitrarily a guess displacement field on the interface linking the body part and the plate part, we first compute the resulting displacement field in the body part by solving a certain three-dimensional purely elastic problem which governs the deformation of the body part. Thus the stress field related to this displacement field is available on the interface. We next compute the displacement field on the plate part by solving purely a plate problem which governs the deformation of the plate part, with the aforementioned stress field as the extra "applied force" on the interface. Now we have two displacement fields on the interface, the original guess displacement and the one just obtained by computing the plate problem. We can make a weighted average of the two to get a new displacement field on the interface. We then use it as a new guess displacement field on the interface to implement the previous process again. Repeat the iteration until the desired result is achieved.

After obtaining the continuous version of the method, we can easily get the discrete algorithm in the same manner. For convenience of presentation, it is described in variational form.

D-F Alternating Algorithm. Let $\vec{\lambda}_{h}^{0} \in\left(V_{h}^{1}\left(\omega_{b}\right)\right)^{2} \times V_{h}^{2}\left(\omega_{b}\right)$ be any given vector-valued function, and $\theta \in(0,1)$ a fixed parameter. Then the vector-valued function sequences $\left\{\vec{\lambda}_{h}^{n}\right\},\left\{\vec{u}_{h}^{\omega, n}\right\}$ and $\left\{\vec{u}_{h}^{\Omega, n}\right\}$ are formed as follows: 
Step 0. $n=0$. Solve the problems

$$
\begin{aligned}
& \left\{\begin{array}{l}
\vec{u}_{h}^{\Omega, n+1} \in\left(V_{h}^{1}(\Omega)\right)^{3}, \\
\vec{u}_{h}^{\Omega, n+1}(Q)=\vec{\lambda}_{h}^{n}(Q), \quad \forall \operatorname{nodes} Q \in \omega_{b}, \\
D_{\Omega, h}\left(\vec{u}_{h}^{\Omega, n+1}, \vec{v}^{\Omega}\right)=F_{\Omega}\left(\vec{v}^{\Omega}\right), \quad \forall \vec{v}^{\Omega} \in\left(V_{h}^{1}\left(\Omega ; \omega_{b}\right)\right)^{3},
\end{array}\right. \\
& \left\{\begin{array}{l}
u_{h}^{\omega, n+1} \in\left(V_{h}^{1}\left(\omega ; \gamma_{0}\right)\right)^{2} \times V_{h}^{2}\left(\omega ; \gamma_{0}\right), \\
D_{\omega, h}\left(\vec{u}_{h}^{\omega, n+1}, \vec{v}^{\omega}\right)=F_{\omega}\left(\vec{v}^{\omega}\right)+\left\{F_{\Omega}\left(\vec{v}^{\Omega}\right)-D_{\Omega, h}\left(\vec{u}_{h}^{\Omega, n+1}, \vec{v}^{\Omega}\right)\right\}
\end{array}\right.
\end{aligned}
$$

where $\vec{v}^{\omega} \in\left(V_{h}^{1}\left(\omega ; \gamma_{0}\right)\right)^{2} \times V_{h}^{2}\left(\omega ; \gamma_{0}\right)$ and $\vec{v}^{\Omega} \in\left(V_{h}^{1}(\Omega)\right)^{3}$ satisfy that $\vec{v}^{\Omega}(Q)=\vec{v}^{\omega}(Q), \forall$ nodes $Q \in \omega_{b}$. Set

$$
\vec{\lambda}_{h}^{n+1}=\theta \vec{\lambda}_{h}^{n}+\left.(1-\theta) \vec{u}_{h}^{\omega, n+1}\right|_{\omega_{b}}
$$

Step $n . n:=n+1$. Repeat the above iteration until achieving the desired accuracy.

Now we give the convergence rate estimate for the D-F alternating algorithm.

Let $\vec{\mu}_{h}^{n}:=\vec{\lambda}_{h}^{n}-\left.\vec{u}_{h}^{\omega}\right|_{\omega_{b}}, \vec{\delta}_{h}^{\omega, n}:=\vec{u}_{h}^{\omega, n}-\vec{u}_{h}^{\omega}, \vec{\delta}_{h}^{\Omega, n}:=\vec{u}_{h}^{\Omega, n}-\vec{u}_{h}^{\Omega}$. Then it follows from (2.8), (4.11)-4.13) that

$$
\begin{aligned}
& \left\{\begin{array}{l}
\vec{\delta}_{h}^{\Omega, n+1} \in\left(V_{h}^{1}(\Omega)\right)^{3}, \\
\vec{\delta}_{h}^{\Omega, n+1}(Q)=\vec{\mu}_{h}^{n}(Q), \quad \forall \operatorname{nodes} Q \in \omega_{b}, \\
D_{\Omega, h}\left(\vec{\delta}_{h}^{\Omega, n+1}, \vec{v}^{\Omega}\right)=0, \quad \forall \vec{v}^{\Omega} \in\left(V_{h}^{1}\left(\Omega ; \omega_{b}\right)\right)^{3},
\end{array}\right. \\
& \left\{\begin{array}{l}
\vec{\delta}_{h}^{\omega, n+1} \in\left(V_{h}^{1}\left(\omega ; \gamma_{0}\right)\right)^{2} \times V_{h}^{2}\left(\omega ; \gamma_{0}\right), \\
D_{\omega, h}\left(\vec{\delta}_{h}^{\omega, n+1}, \vec{v}^{\omega}\right)+D_{\Omega, h}\left(\vec{\delta}_{h}^{\Omega, n+1}, \vec{v}^{\Omega}\right)=0,
\end{array}\right.
\end{aligned}
$$

and

$$
\tilde{\mu}_{h}^{n+1}=\theta \tilde{\mu}_{h}^{n}+(1-\theta) \vec{\delta}_{h}^{\omega, n+1},
$$

where $\vec{v}^{\omega}$ and $\vec{v}^{\Omega}$ are defined as before, and $\tilde{\mu}_{h}^{n}$ denotes the extension of the vectorvalued function $\vec{\mu}_{h}^{n}$ on $\omega$ such that

$$
\left\{\begin{array}{l}
D_{\omega, h}\left(\tilde{\mu}_{h}^{n}, \vec{v}^{\omega}\right)=0, \quad \forall \vec{v}^{\omega} \in\left(V_{h}^{1}\left(\omega_{c}\right)\right)^{2} \times V_{h}^{2}\left(\omega_{c}\right) \\
\quad \text { with } \vec{v}^{\omega}(Q)=\partial_{\alpha} v_{3}^{\omega}(Q)=0, Q \in \gamma_{0} \cup \gamma_{1}, \\
\tilde{\mu}_{h}^{n}=\vec{\mu}_{h}^{n} \quad \text { on } \omega_{b} .
\end{array}\right.
$$

We then have the following result.

Theorem 2. There exists a fixed parameter $\theta^{*} \in(0,1)$, independent of the mesh size $h$, such that for the $D-F$ alternating algorithm with the parameter $\theta=\theta^{*}$ we have the estimates

$$
\begin{aligned}
& \left\|\vec{\mu}_{h}^{n}\right\|_{h} \leq C \rho^{* n}\left\|\vec{\mu}_{h}^{0}\right\|_{h}, \\
& D_{\Omega, h}\left(\vec{\delta}_{h}^{\Omega, n}, \vec{\delta}_{h}^{\Omega, n}\right)+D_{\omega, h}\left(\vec{\delta}_{h}^{\omega, n}, \vec{\delta}_{h}^{\omega, n}\right) \leq C \rho^{* 2 n}\left\|\vec{\mu}_{h}^{0}\right\|_{h}^{2},
\end{aligned}
$$

where $\rho^{*}=\sqrt{\theta^{*}}$, and for $\vec{v}:=\left(v_{i}\right) \in\left(V_{h}^{1}\left(\omega_{b}\right)\right)^{2} \times V_{h}^{2}\left(\omega_{b}\right)$,

$$
\|\vec{v}\|_{h}:=\left(\sum_{i=1}^{2}\left\|v_{i}\right\|_{\frac{1}{2}, \omega_{b}}^{2}+\left\|v_{3}\right\|_{2, h, \omega_{b}}^{2}\right)^{1 / 2} .
$$

Proof. We have from (4.16) that

$$
\begin{aligned}
D_{\omega, h}\left(\tilde{\mu}_{h}^{n+1}, \tilde{\mu}_{h}^{n+1}\right)= & \theta^{2} D_{\omega, h}\left(\tilde{\mu}_{h}^{n}, \tilde{\mu}_{h}^{n}\right)+2 \theta(1-\theta) D_{\omega, h}\left(\tilde{\mu}_{h}^{n}, \vec{\delta}_{h}^{\omega, n+1}\right) \\
& +(1-\theta)^{2} D_{\omega, h}\left(\vec{\delta}_{h}^{\omega, n+1}, \vec{\delta}_{h}^{\omega, n+1}\right) .
\end{aligned}
$$


It follows from (4.14) that

$$
\vec{\delta}_{h}^{\Omega, n+1}(Q)=\vec{\mu}_{h}^{n}(Q), \forall \text { nodes } Q \in \omega_{b} .
$$

Therefore we can choose $\vec{v}^{\omega}=\tilde{\mu}_{h}^{n}, \vec{v}^{\Omega}=\vec{\delta}_{h}^{\Omega, n+1}$ in 4.15) to get

$$
D_{\omega, h}\left(\tilde{\mu}_{h}^{n}, \vec{\delta}_{h}^{\omega, n+1}\right)=-D_{\Omega, h}\left(\vec{\delta}_{h}^{\Omega, n+1}, \vec{\delta}_{h}^{\Omega, n+1}\right) \geq 0 .
$$

Since $\vec{\delta}_{h}^{\omega, n+1}, \vec{\delta}_{h}^{\Omega, n+1}$ satisfy (4.14) and (4.15), it follows from Lemma 5 that (the constant $C^{*}$ is the one in Lemma (4)

$$
D_{\omega, h}\left(\vec{\delta}_{h}^{\omega, n+1}, \vec{\delta}_{h}^{\omega, n+1}\right) \leq C^{*} D_{\Omega, h}\left(\vec{\delta}_{h}^{\Omega, n+1}, \vec{\delta}_{h}^{\Omega, n+1}\right),
$$

which together with (4.19) yields

$$
\begin{aligned}
D_{\Omega, h}\left(\vec{\delta}_{h}^{\Omega, n+1}, \vec{\delta}_{h}^{\Omega, n+1}\right) & \leq D_{\omega, h}^{1 / 2}\left(\tilde{\mu}_{h}^{n}, \tilde{\mu}_{h}^{n}\right) D_{\omega, h}^{1 / 2}\left(\vec{\delta}_{h}^{\omega, n+1}, \vec{\delta}_{h}^{\omega, n+1}\right) \\
& \leq \sqrt{C^{*}} D_{\omega, h}^{1 / 2}\left(\tilde{\mu}_{h}^{n}, \tilde{\mu}_{h}^{n}\right) D_{\Omega, h}^{1 / 2}\left(\vec{\delta}_{h}^{\Omega, n+1}, \vec{\delta}_{h}^{\Omega, n+1}\right),
\end{aligned}
$$

that is,

$$
D_{\omega, h}\left(\vec{\delta}_{h}^{\omega, n+1}, \vec{\delta}_{h}^{\omega, n+1}\right) \leq C^{*} D_{\Omega, h}\left(\vec{\delta}_{h}^{\Omega, n+1}, \vec{\delta}_{h}^{\Omega, n+1}\right) \leq C^{* 2} D_{\omega, h}\left(\tilde{\mu}_{h}^{n}, \tilde{\mu}_{h}^{n}\right) .
$$

Therefore it follows from (4.18)-(4.20) that

$$
D_{\omega, h}\left(\tilde{\mu}_{h}^{n+1}, \tilde{\mu}_{h}^{n+1}\right) \leq\left[\theta^{2}+C^{* 2}(1-\theta)^{2}\right] D_{\omega, h}\left(\tilde{\mu}_{h}^{n}, \tilde{\mu}_{h}^{n}\right) .
$$

Now taking $\theta=\theta^{*}=\frac{C^{* 2}}{1+C^{* 2}} \in(0,1)$ in the above inequality, we have

$$
D_{\omega, h}\left(\tilde{\mu}_{h}^{n+1}, \tilde{\mu}_{h}^{n+1}\right) \leq \rho^{* 2} D_{\omega, h}\left(\tilde{\mu}_{h}^{n}, \tilde{\mu}_{h}^{n}\right),
$$

where $\rho^{*}=\sqrt{\frac{C^{* 2}}{1+C^{* 2}}}=\sqrt{\theta^{*}}$, which immediately implies

$$
D_{\omega, h}\left(\tilde{\mu}_{h}^{n}, \tilde{\mu}_{h}^{n}\right) \leq \rho^{* 2 n} D_{\omega, h}\left(\tilde{\mu}_{h}^{0}, \tilde{\mu}_{h}^{0}\right) .
$$

Furthermore, arguing as in the proof of Lemma 3, we have

$$
C_{1}\left\|\vec{\mu}_{h}^{n}\right\|_{h}^{2} \leq D_{\omega, h}\left(\tilde{\mu}_{h}^{n}, \tilde{\mu}_{h}^{n}\right) \leq C_{2}\left\|\vec{\mu}_{h}^{n}\right\|_{h}^{2} .
$$

This with 4.21 gives

$$
\left\|\vec{\mu}_{h}^{n}\right\|_{h} \leq C \rho^{* n}\left\|\vec{\mu}_{h}^{0}\right\|_{h} .
$$

The other inequality of (4.17) follows from (4.19), (4.22) and Lemma 5 in a similar manner.

According to Theorem 2, it is of practical importance to choose the parameter $\theta^{*} \in(0,1)$ reasonably, or equivalently, to give the upper bound estimate for the constant $C^{*}$ in Lemma 4, to guarantee the convergence of the D-F alternating algorithm. One simple way is that, since the inequality (3.20) naturally holds when $C^{*}$ is chosen large enough, $\theta^{*}$ can then be taken as a positive number very close to 1 (but strictly less than 1). Or alternatively, we can first execute the D-F alternating algorithm with a positive number $\theta_{1} \in\left(\frac{1}{2}, 1\right)$. If the computational results behave badly, then replace $\theta_{1}$ by the other number $\theta_{2} \in\left(\theta_{1}, 1\right)$ and execute the $\mathrm{D}-\mathrm{F}$ alternating algorithm again. We may repeat this process several times to get the desired parameter $\theta^{*}$. Obviously, more numerical experience is needed to use this method in a more sophisticated way. 
The other way is to compute $C^{*}$ in Lemma 4 by the power method (cf. [16]). That means we want to estimate the term

$$
\sup \frac{D_{\Omega, h}\left(\left[\vec{v}^{\omega}\right]^{\Omega},\left[\vec{v}^{\omega}\right]^{\Omega}\right)}{D_{\omega, h}\left(\vec{v}^{\omega}, \vec{v}^{\omega}\right)}
$$

with the supremum taken over the set $\left(V_{h}^{1}\left(\omega ; \gamma_{0}\right)\right)^{2} \times V_{h}^{2}\left(\omega ; \gamma_{0}\right) \backslash\{0\}$. For clarity we attempt to rewrite (4.23) in matrix form. We first divide the index set $I_{2}$ given before into two subsets, i.e., $I_{2}=I_{2 b} \cup I_{2 c}$, where $I_{2 b}$ consists of the indices of the nodal parameters related to the finite element spaces $\left(V_{h}^{1}(\Omega)\right)^{3}$ and with nodes in $\bar{\omega}_{b}$, and $I_{2 c}:=I_{2} \backslash I_{2 b}$. For $\vec{v}^{\omega} \in\left(V_{h}^{1}\left(\omega ; \gamma_{0}\right)\right)^{2} \times V_{h}^{2}\left(\omega ; \gamma_{0}\right)$, let $\bar{v}_{2}:=\left(\bar{v}_{2 b}, \bar{v}_{2 c}\right)$ denote its nodal parameter representation partitioned into two parts via the index sets $I_{2 b}$ and $I_{2 c}$. Therefore by definition (3.9) we have

$$
D_{\Omega, h}\left(\left[\vec{v}^{\omega}\right]^{\Omega},\left[\vec{v}^{\omega}\right]^{\Omega}\right)=\left[\bar{S}_{1} \bar{v}_{2 b}, \bar{v}_{2 b}\right],
$$

where $\bar{S}_{1}$ is the Schur complement associated with the bilinear form $D_{\Omega, h}(\cdot, \cdot)$ defined on the set of functions in $\left(V_{h}^{1}(\Omega)\right)^{3}$ with the prescribed nodal parameters $\bar{v}_{2 b}$ on $\omega_{b}$ and with the free boundary conditions on $\partial \Omega \backslash \bar{\omega}$. Similarly,

$$
D_{\omega, h}\left(\vec{v}^{\omega}, \vec{v}^{\omega}\right)=\left[\bar{T}_{2} \bar{v}_{2}, \bar{v}_{2}\right],
$$

where $\bar{T}_{2}$ is the stiffness matrix associated with the bilinear form $D_{\omega, h}(\cdot, \cdot)$. Then (4.23) amounts to

$$
\sup _{\bar{v}_{2} \neq 0} \frac{\left[\bar{S}_{1} \bar{v}_{2 b}, \bar{v}_{2 b}\right]}{\left[\bar{T}_{2} \bar{v}_{2}, \bar{v}_{2}\right]}=\lambda_{\max }\left(\bar{T}_{2}^{-\frac{1}{2}}\left[\begin{array}{ll}
\bar{S}_{1} & \\
& 0
\end{array}\right] \bar{T}_{2}^{-\frac{1}{2}}\right) .
$$

Hence the approximate vector sequence constructed for computing the eigenvector related to the maximal eigenvalue of $\bar{T}_{2}^{-\frac{1}{2}}\left[\begin{array}{ll}\bar{S}_{1} & \\ & 0\end{array}\right] \bar{T}_{2}^{-\frac{1}{2}}$ is $\left(x_{0}\right.$ denotes the initial vector)

$$
\left(\bar{T}_{2}^{-\frac{1}{2}}\left[\begin{array}{ll}
\bar{S}_{1} & \\
& 0
\end{array}\right] \bar{T}_{2}^{-\frac{1}{2}}\right)^{n} x_{0},
$$

which, together with the Rayleigh quotient representation, yields a sequence for computing the maximal eigenvalue of $\bar{T}_{2}^{-\frac{1}{2}}\left[\begin{array}{ll}\bar{S}_{1} & \\ & 0\end{array}\right] \bar{T}_{2}^{-\frac{1}{2}}$, given by

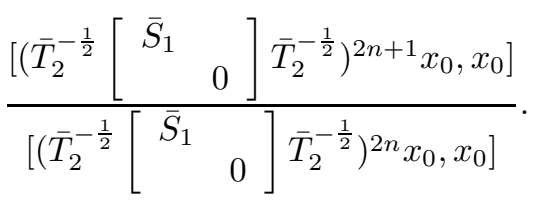

Noting that

$$
\left(\bar{T}_{2}^{-\frac{1}{2}}\left[\begin{array}{ll}
\bar{S}_{1} & \\
& 0
\end{array}\right] \bar{T}_{2}^{-\frac{1}{2}}\right)^{n}=\bar{T}_{2}^{\frac{1}{2}}\left(\bar{T}_{2}^{-1}\left[\begin{array}{ll}
\bar{S}_{1} & \\
& 0
\end{array}\right]\right)^{n} \bar{T}_{2}^{-\frac{1}{2}},
$$

we then have the following algorithm for computing $\lambda_{\max }\left(\bar{T}_{2}^{-\frac{1}{2}}\left[\begin{array}{ll}\bar{S}_{1} & \\ & 0\end{array}\right] \bar{T}_{2}^{-\frac{1}{2}}\right)$. 
Step 0 . Given initial the vector $z_{0} \neq 0$, set $n=0$.

Step $n$. Compute $z_{2 n+1}$ and $z_{2 n+2}$ by $\bar{T}_{2} z_{2 n+1}=\left[\begin{array}{ll}\bar{S}_{1} & \\ & 0\end{array}\right] z_{n}$ and $\bar{T}_{2} z_{2 n+2}=$ $\left[\begin{array}{ll}\bar{S}_{1} & \\ & 0\end{array}\right] z_{n+1}$. Set $\hat{z}_{2 n+2}=\frac{z_{2 n+2}}{\max \left(z_{2 n+2}\right)}$ and compute $\alpha_{n}$ by the formula $\alpha_{n}:=\frac{\left[\hat{z}_{2 n+2},\left[\begin{array}{ll}\bar{S}_{1} & \\ & 0\end{array}\right] z_{0}\right]}{\left[\hat{z}_{2 n+2}, \bar{T}_{2} z_{0}\right]}$.

Set $n:=n+1$. We repeat the above iteration until we obtain the desired result.

The main computational work of this algorithm is the numerical solutions of the pure elastic body problem and the pure elastic plate problem, which can be solved by existing efficient algorithms.

Finally we give some comparisons between the two methods presented above. A common feature of the two methods is that, at each iteration step, one needs only to solve either a pure body or a pure plate problem, which can be realized by many known efficient numerical solvers. Therefore, both methods reflect the essence of elastic multi-structures, that is, elastic multi-structures are complicated globally but their substructures are rather simple locally. On the other hand, there are some differences between the two methods. The first method needs to form the global stiffness matrix related to the elastic body-plate problem for the use of the preconditioned conjugate gradient method. The second one does not need to do so, but it requires the choice of a proper parameter to guarantee convergence.

\section{ACKNOWLEDGMENTS}

The author is very grateful to an anonymous referee, whose careful examination and constructive suggestions greatly improved this paper. The author is also grateful to Prof. Zhongci Shi and Prof. Jun Zou, who improved the English of this paper.

\section{REFERENCES}

[1] I. Babuska and A. Aziz, Survey lectures on the mathematical foundations of the finite element method, in The Mathematical Foundations of the Finite Element Method with Applications to Partial Differential Equations, A. K. Aziz eds., Academic Press, New York and London, 1972. MR 54:9111

[2] M. Bernadou, S. Fayolle and F. Lene, Numerical analysis of junctions between plates, Comput. Methods Appl. Mech. Engrg., 74(1989), pp. 307-326. MR 90j:73062

[3] P. E. Bjørstad and O. B. Widlund, Iterative method for the solution of elliptic problems on regions partitioned into substructures, SIAM J. Numer. Anal., 23(1986), pp. 1097-1120. MR 88h:65188

[4] H. Blum and R. Rannacher, On the boundary value problem of the biharmonic operators on domains with angular corners, Math. Meth. Appl. Sci., 2(1980), pp. 556-581. MR 82a:35022

[5] J. H. Bramble, J. E. Pasciak and A. H. Schatz, An iterative method for elliptic problems on regions partitioned into substructures, Math. Comp., 46(1986), pp. 361-369. MR 88a:65123

[6] J. H. Bramble, J. E. Pasciak and A. H. Schatz, The construction of preconditioners for elliptic problems by substructuring, I, Math. Comp., 47(1986), pp. 103-134. MR 87m:65174

[7] S. C. Brenner, A two-level Schwarz preconditioner for nonconforming plate elements, in Domain Decomposition Methods in Scientific Computing (Proceedings of DDM 7) eds. David E. Keyes and Jinchao Xu, 1994, Contemp. Math. 180, pp. 9-14. MR 95j:65134 
[8] S. C. Brenner, Two-level additive Schwarz preconditioners for nonconforming finite element methods, Math. Comp., 65(1996), pp. 897-921. MR 96j:65117

[9] S. C. Brenner, A two-level additive Schwarz preconditioner for nonconforming plate elements, Numer. Math., 72(1996), pp. 419-447. MR 97h:65147

[10] T. F. Chan and T. Mathew, Domain decomposition algorithms, Acta Numerica, 2(1994), pp. 61-143. MR 95f:65214

[11] P. G. Ciarlet, The Finite Element Method for Elliptic Problems, North-Holland, Amsterdam, 1978. MR 58:25001

[12] P. G. Ciarlet, Plate and Junctions in Elastic Multi-structures, Springer-Verlag, BerlinHeiderberg-New York, 1990. MR 91h:73004

[13] M. Dryja and O. B. Widlund, Towards a unified theory of domain decomposition algorithms for elliptic problem, in Third International Symposium on Domain Decomposition Methods for PDE (eds T. F. Chan et al), SIAM, Philadelphia, 1990, 3-21 MR 91m:65294

[14] K. Feng, Elliptic equations on composite manifold and composite elastic structures (in Chinese), Math. Numer. Sinica, 1(1979), pp. 199-208. MR 83m:49041

[15] K. Feng and Z. Shi, Mathematical Theory of Elastic Structures (in Chinese), Science Presss, Beijing, 1981. (English translation, Springer-Verlag \& Science Press, Berlin-New York, 1995.) MR 84f:73001 MR 97b:73001

[16] G. H. Golub and C. F. Van Loan, Matrix computation (2nd edn)., The Johns Hopkins University Press, Baltimore, 1989. MR 90d:65055

[17] P. G. Grisvard, Singularities in Boundary Value Problems, Springer-Verlag, BerlinHeidelberg-New York, 1992. MR 93h:35004

[18] F. d'Hennezel, Domain decomposition method and elastic multi-structures: the stiffened plate problem, Numer. Math., 66(1993), pp. 181-197. MR 95c:73080

[19] L. D. Marini and A. Quarteroni, A relaxation procedure for domain decomposition methods using finite elements, Numer. Math., 55(1989), pp. 575-598. MR 90g:65150

[20] J. Necăs, Les Méthodes Directes en Théorie des Équations Elliptiques, Masson, Paris, 1967. MR 37:3168

[21] A. Quarteroni, G. S. Landriani and A. Valli, Coupling of viscous and inviscid Stokes equations via a domain decomposition method for finite elements, Numer. Math., 59(1991), pp. 831-859. MR 93b:65190

[22] F. Stummel, The generalized patch test, SIAM J. Numer. Anal., 16(1979), pp. 199-208. MR 80e:65106

[23] F. Stummel, Basic compactness properties of nonconforming and hybrid finite element spaces, RAIRO Anal. Numer., 4(1980), pp. 81-115. MR 81h:65058

[24] L. Wang, The mathematical models of some composite elastic structures and their finite element approximations (in Chinese), Numer. Math. Sinica, 14(1992), pp. 358-370.

[25] L. Wang, An analysis of the TRUNC elements for coupled plates with rigid junction, Comput. Methods Appl. Mech. Engrig., 121(1995), pp. 1-14. MR 96b:73105

[26] J. Xu, Theory of multilevel methods, Ph. D. thesis, Cornell University, 1989.

[27] J. Xu and J. Zou, Non-overlapping domain decomposition methods, SIAM Review, 40(1998), pp. 857-914. MR 99m:65241

Department of Mathematics, Shanghai Jiao Tong University, Shanghai, 200240, PeoPles Republic of China

E-mail address: jghuang@online.sh.cn 